\title{
TOWARDS A COMBINED FRACTIONAL MECHANICS AND QUANTIZATION
}

\author{
Agnieszka B. Malinowska ${ }^{1}$, Delfim F. M. Torres ${ }^{2}$
}

\begin{abstract}
A fractional Hamiltonian formalism is introduced for the recent combined fractional calculus of variations. The Hamilton-Jacobi partial differential equation is generalized to be applicable for systems containing combined Caputo fractional derivatives. The obtained results provide tools to carry out the quantization of nonconservative problems through combined fractional canonical equations of Hamilton type.
\end{abstract}

MSC 2010: Primary 26A33, 49K05; Secondary 49S05

Key Words and Phrases: fractional canonical formalism, Hamiltonian approach, variational principles of physics, nonconservative systems, combined fractional derivatives, variational calculus

\section{Introduction}

The conservative physical systems imply frictionless motion and are simplification of the real dynamical world. The systems in Nature always contain internal damping and are subject to some external forces that do not store energy and which are not equivalent to the gradient of a potential. For nonconservative dynamical systems the energy conservation law is broken and, as a consequence, the standard Hamiltonian formalism is no longer valid for describing the behavior of the system. Moreover, not only the energy, but also other physical quantities such as linear or angular momentums, are not conserved: Noether's classical theorem ceases to be valid [11].

In order to model nonconservative dynamical systems, a novel approach to variational calculus was proposed by Riewe in 1996 and 1997, 24, 25.

This is a preprint of a paper whose final and definite form will be published in: Fract. Calc. Appl. Anal., Vol. 15, No 3 (2012). Submitted 21-Feb-2012; revised 29-May-2012; accepted 03-June-2012. 
The idea is based on the concept of fractional differentiation and fractional integration, 2, 9]. Roughly speaking, the appearance of fractional equations is natural due to physical reasons of long-range dissipation, fractional wave propagation in complex media and long memory. Fractionalorder Euler-Lagrange equations and the Hamilton formalism with fractional derivatives are investigated in [16, 23. For a fractional Hamiltonian analysis of irregular systems, see [8].

The fractional calculus of variations is nowadays an emerging field see [4, 6, 7, 9, 10, 17] and references therein. A general fractional variational calculus, based on a combined Caputo derivative, was recently introduced by the authors in [19] and further developed in [20] and [21]. In [3], a generalization of the fractional variational calculus is given by considering generalized Hilfer's fractional derivatives with two and three parameters. Here we study the importance of the new combined variational calculus in fractional mechanics, describing nonconservative dynamic systems.

In Section 2 we recall the combined fractional Euler-Lagrange equations. Our results are then given in Section 3. In Section 3.1 we show that combined fractional Hamiltonian equations of motion can be obtained for nonconservative systems. The constants of motion cease to be valid and a new notion is introduced in Section 3.2. Canonical fractional transformations of first and second kind are studied in Section 3.3 and Section 3.4 , respectively. Subsequently, in Section [3.5, an Hamilton-Jacobi type equation is derived and a fractional quantum wave equation suggested.

The understanding of the Hamiltonian dynamical systems, classical or quantum, has been a long standing theoretical question since Dirac's quantization of the classical electromagnetic field. The combined fractional Hamiltonian approach here introduced seems to be a promising direction of research.

\section{Preliminaries}

Let $\alpha, \beta \in(0,1)$ and $\gamma \in[0,1]$. The fractional derivative operator ${ }^{C} D_{\gamma}^{\alpha, \beta}$ was introduced in Malinowska and Torres [19], as

$$
{ }^{C} D_{\gamma}^{\alpha, \beta}:=\gamma_{a}^{C} D_{x}^{\alpha}+(1-\gamma){ }_{x}^{C} D_{b}^{\beta} .
$$

For $\gamma=0$ and $\gamma=1$ we obtain the standard Caputo operators: ${ }^{C} D_{0}^{\alpha, \beta}=$ ${ }_{x}^{C} D_{b}^{\beta}$ and ${ }^{C} D_{1}^{\alpha, \beta}={ }_{a}^{C} D_{x}^{\alpha}$. Consider a fractional Lagrangian

$$
L\left(t, \mathbf{q},{ }^{C} D_{\gamma}^{\alpha, \beta} \mathbf{q}\right)
$$

depending on time $t$, the coordinates $\mathbf{q}=\left[q_{1}, \ldots, q_{N}\right]$ and their fractional velocities ${ }^{C} D_{\gamma}^{\alpha, \beta} \mathbf{q}=\left[{ }^{C} D_{\gamma_{1}}^{\alpha_{1}, \beta_{1}} q_{1}, \ldots,{ }^{C} D_{\gamma_{N}}^{\alpha_{N}, \beta_{N}} q_{N}\right]$. The Euler-Lagrange 
equations corresponding to (11) were studied in [20] and form the following system of $N$ fractional differential equations:

$$
\frac{\partial L}{\partial q_{i}}+D_{1-\gamma_{i}}^{\beta_{i}, \alpha_{i}} \frac{\partial L}{\partial^{C} D_{\gamma_{i}}^{\alpha_{i}, \beta_{i}} q_{i}}=0, \quad i=1, \ldots N
$$

where

$$
D_{1-\gamma_{i}}^{\beta_{i}, \alpha_{i}}:=\left(1-\gamma_{i}\right)_{a} D_{x}^{\beta_{i}}+\gamma_{i x} D_{b}^{\alpha_{i}}
$$

with ${ }_{a} D_{x}^{\beta_{i}}$ and ${ }_{x} D_{b}^{\alpha_{i}}$ denoting the classical left and right Riemann-Liouville fractional derivatives.

REMARK 2.1. With the notation from [20, 21],

$$
\partial_{i+1} L=\frac{\partial L}{\partial q_{i}} \text { and } \partial_{N+1+i} L=\frac{\partial L}{\partial^{C} D_{\gamma_{i}}^{\alpha_{i}, \beta_{i}} q_{i}}, \quad i=1, \ldots N .
$$

In contrast with [20, 21], we adopt here the notation used in mechanics [14, 25].

More general necessary optimality conditions than (2) are found in [20, 21]. However, all our previous works [19, 20, 21] on combined fractional derivatives consider the Lagrangian approach only. In contrast, here we develop the combined fractional Hamiltonian formalism. We show that (2) can be reduced to a special Hamiltonian system of $2 N$ fractional differential equations: the combined canonical fractional equations.

\section{Main Results}

In analogy with the classical mechanics, let us introduce the canonical momenta $p_{i}$ by

$$
p_{i}=\frac{\partial L}{\partial^{C} D_{\gamma_{i}}^{\alpha_{i}, \beta_{i}} q_{i}}, \quad i=1, \ldots N
$$

\subsection{Combined Euler-Lagrange equations in Hamiltonian form}

Assume that $\left|\frac{\partial\left(p_{1}, \ldots, p_{N}\right)}{\partial\left({ }^{C} D_{\gamma_{1}}^{\alpha_{1}, \beta_{1}} q_{1}, \ldots,{ }^{C} D_{\gamma_{N}}^{\alpha_{N}, \beta_{N}} q_{N}\right)}\right| \neq 0$. Then, by the implicit function theorem, we can locally solve equations (3) with respect to ${ }^{C} D_{\gamma_{1}}^{\alpha_{1}, \beta_{1}} q_{1}, \ldots,{ }^{C} D_{\gamma_{N}}^{\alpha_{N}, \beta_{N}} q_{N}$. The fractional Hamiltonian is defined by

$$
H(t, \mathbf{q}, \mathbf{p})=\sum_{i=1}^{N} p_{i}{ }^{C} D_{\gamma_{i}}^{\alpha_{i}, \beta_{i}} q_{i}-L\left(t, \mathbf{q},{ }^{C} D_{\gamma}^{\alpha, \beta} \mathbf{q}\right)
$$


where ${ }^{C} D_{\gamma_{i}}^{\alpha_{i}, \beta_{i}} q_{i}$ are regarded as functions of variables $t, q_{1}, \ldots, q_{N}, p_{1}, \ldots$, $p_{N}$. Therefore, the differential of $H$ is given by

$$
d H=\sum_{i=1}^{N} \frac{\partial H}{\partial q_{i}} d q_{i}+\sum_{i=1}^{N} \frac{\partial H}{\partial p_{i}} d p_{i}+\frac{\partial H}{\partial t} d t .
$$

From the defining equality (4), we can also write

$$
\begin{aligned}
d H=\sum_{i=1}^{N}{ }^{C} D_{\gamma_{i}}^{\alpha_{i}, \beta_{i}} q_{i} d p_{i}+\sum_{i=1}^{N} p_{i} d^{C} D_{\gamma_{i}}^{\alpha_{i}, \beta_{i}} q_{i}-\sum_{i=1}^{N} \frac{\partial L}{\partial q_{i}} d q_{i} \\
\quad-\sum_{i=1}^{N} \frac{\partial L}{\partial^{C} D_{\gamma_{i}}^{\alpha_{i}, \beta_{i}} q_{i}} d^{C} D_{\gamma_{i}}^{\alpha_{i}, \beta_{i}} q_{i}-\frac{\partial L}{\partial t} d t .
\end{aligned}
$$

The terms containing $d^{C} D_{\gamma_{i}}^{\alpha_{i}, \beta_{i}} q_{i}$ in (6) cancel because of the definition of canonical momenta. Applying relations (2), we get

$$
d H=\sum_{i=1}^{N}{ }^{C} D_{\gamma_{i}}^{\alpha_{i}, \beta_{i}} q_{i} d p_{i}+\sum_{i=1}^{N} D_{1-\gamma_{i}}^{\beta_{i}, \alpha_{i}} p_{i} d q_{i}-\frac{\partial L}{\partial t} d t .
$$

Comparison with (5) furnishes the following set of $2 N$ relations:

$$
\frac{\partial H}{\partial p_{i}}={ }^{C} D_{\gamma_{i}}^{\alpha_{i}, \beta_{i}} q_{i}, \quad \frac{\partial H}{\partial q_{i}}=D_{1-\gamma_{i}}^{\beta_{i}, \alpha_{i}} p_{i}, \quad i=1, \ldots, N,
$$

which we can call the combined fractional canonical equations of Hamilton. They constitute a set of $2 N$ fractional order equations of motion replacing the Euler-Lagrange equations (2). Moreover,

$$
\frac{\partial H}{\partial t}=-\frac{\partial L}{\partial t} \text {. }
$$

For integer-order derivatives, it can be shown that

$$
\frac{d H}{d t}=\frac{\partial H}{\partial t}=-\frac{\partial L}{\partial t}
$$

(see, e.g., [14, p. 220]). Hence, if $L$ (and, in consequence of (8), also $H$ ) is not an explicit function of $t$, i.e., in the autonomous case, then $H$ is a constant of motion. This is a well-known consequence of Noether's theorem [27, 29]. With noninteger-order derivatives this is not the case, see [12, 13]. Observe that using equations (7), we can write

$$
\frac{d H}{d t}=\sum_{i=1}^{N}\left({ }^{C} D_{\gamma_{i}}^{\alpha_{i}, \beta_{i}} q_{i} \frac{d p_{i}}{d t}+D_{1-\gamma_{i}}^{\beta_{i}, \alpha_{i}} p_{i} \frac{d q_{i}}{d t}\right)+\frac{\partial H}{\partial t} .
$$

In contrast with the integer-order case, the terms containing fractional and classical derivatives of coordinates and momenta do not cancel. Therefore, 
in general we obtain nonconservative systems and classical constants of motion cease to be valid [11, 24. To deal with the problem we introduce the notion of fractional constant of motion.

\subsection{Fractional constants of motion}

To account for the presence of dissipative terms, we propose the following definition of fractional constant of motion.

Definition 3.1. We say that a function $C(t, \mathbf{q}, \mathbf{p})$ is a fractional constant of motion of order $(\alpha, \beta, \gamma)$ if $D_{1-\gamma}^{\beta, \alpha}[t \mapsto C(t, \mathbf{q}(t), \mathbf{p}(t))]$ is the null function along any pair $(\mathbf{q}, \mathbf{p})$ satisfying the $2 N$ combined fractional canonical equations (7).

It follows from (77) that if $q_{i}$ is absent in the fractional Hamiltonian, then $D_{1-\gamma_{i}}^{\beta_{i}, \alpha_{i}} p_{i}=0$, i.e., if $\frac{\partial L}{\partial q_{i}}=0$, then $p_{i}=\frac{\partial L}{\partial^{C} D_{\gamma_{i}}^{\alpha_{i}, \beta_{i}} q_{i}}$ is a fractional constant of motion of order $\left(\alpha_{i}, \beta_{i}, \gamma_{i}\right)$.

\subsection{Canonical fractional transformations of first kind}

We now look for transformations under which the combined fractional canonical equations of Hamilton (7) preserve their canonical form. Consider the simultaneous transformation of independent coordinates and momenta, $q_{i}$ and $p_{i}$, to a new set $Q_{i}$ and $P_{i}$ with transformation equations

$$
Q_{i}=Q_{i}(t, \mathbf{q}, \mathbf{p}), \quad P_{i}=P_{i}(t, \mathbf{q}, \mathbf{p}), \quad i=1, \ldots, N .
$$

The new $Q_{i}$ and $P_{i}$ are canonical coordinates provided there exists some function $K(t, \mathbf{Q}, \mathbf{P})$ such that the equations of motion in the new set are in Hamiltonian form:

$$
\frac{\partial K}{\partial P_{i}}={ }^{C} D_{\gamma_{i}}^{\alpha_{i}, \beta_{i}} Q_{i}, \quad \frac{\partial K}{\partial Q_{i}}=D_{1-\gamma_{i}}^{\beta_{i}, \alpha_{i}} P_{i}, \quad i=1, \ldots, N .
$$

Transformations for which equations (9) are valid are said to be canonical. Function $K$ plays the role of the Hamiltonian in the new coordinates set. If $Q_{i}$ and $P_{i}$ are to be canonical coordinates, then they must satisfy the modified fractional Hamiltonian principle of form

$$
\delta \int_{a}^{b}\left(\sum_{i=1}^{N} P_{i}^{C} D_{\gamma_{i}}^{\alpha_{i}, \beta_{i}} Q_{i}-K(t, \mathbf{Q}, \mathbf{P})\right) d t=0 .
$$

At the same time, the original coordinates satisfy the similar principle

$$
\delta \int_{a}^{b}\left(\sum_{i=1}^{N} p_{i}{ }^{C} D_{\gamma_{i}}^{\alpha_{i}, \beta_{i}} q_{i}-H(t, \mathbf{q}, \mathbf{p})\right) d t=0 .
$$


The simultaneous validity of equations (10) and (11) means that integrands of the two integrals can differ at most by a total derivative of an arbitrary function $F$, sometimes called a gauge term [28]:

$$
\frac{d}{d t} F+\sum_{i=1}^{N} P_{i}^{C} D_{\gamma_{i}}^{\alpha_{i}, \beta_{i}} Q_{i}-K(t, \mathbf{Q}, \mathbf{P})=\sum_{i=1}^{N} p_{i}{ }^{C} D_{\gamma_{i}}^{\alpha_{i}, \beta_{i}} q_{i}-H(t, \mathbf{q}, \mathbf{p}) .
$$

The arbitrary function $F$ works as a generating function of the transformation, and is very useful in developing direct methods to solve problems of the calculus of variations [5, 18] and optimal control [26, 30]. Function $F$ is only specified up to an additive constant. In order to produce transformations between the two sets of canonical variables, $F$ must be a function of both old and new variables. For mechanics involving fractional derivatives (cf. [24]) we need to introduce variables $\bar{q}_{i}$ and $\bar{Q}_{i}, i=1, \ldots, N$, satisfying

$$
\frac{d \bar{q}_{i}}{d t}={ }^{C} D_{\gamma_{i}}^{\alpha_{i}, \beta_{i}} q_{i}, \quad \frac{d \bar{Q}_{i}}{d t}={ }^{C} D_{\gamma_{i}}^{\alpha_{i}, \beta_{i}} Q_{i} .
$$

For $\gamma_{i}=1, \alpha_{i}=1, \beta_{i}=0, i=1, \ldots, N$, these new coordinates are the same as the usual canonical coordinates. However, when dealing with fractional derivatives, the coordinates $\bar{q}_{i}$ and $\bar{Q}_{i}$ will not be canonical, so all canonical expressions must be written in terms of ${ }^{C} D_{\gamma_{i}}^{\alpha_{i}, \beta_{i}} q_{i}$ and ${ }^{C} D_{\gamma_{i}}^{\alpha_{i}, \beta_{i}} Q_{i}$. For a generating function $F_{1}(t, \overline{\mathbf{q}}, \overline{\mathbf{Q}})$, the integrands of (10) and (111) are connected by the relation

$$
\frac{d}{d t} F_{1}(t, \overline{\mathbf{q}}, \overline{\mathbf{Q}})=\sum_{i=1}^{N} p_{i}{ }^{C} D_{\gamma_{i}}^{\alpha_{i}, \beta_{i}} q_{i}-H(t, \mathbf{q}, \mathbf{p})-\sum_{i=1}^{N} P_{i}{ }^{C} D_{\gamma_{i}}^{\alpha_{i}, \beta_{i}} Q_{i}+K(t, \mathbf{Q}, \mathbf{P})
$$

Because

$$
d F_{1}=\sum_{i=1}^{N} \frac{\partial F_{1}}{\partial \bar{q}_{i}} d \bar{q}_{i}+\sum_{i=1}^{N} P_{i} \frac{\partial F_{1}}{\partial \bar{Q}_{i}} d \bar{p}_{i}+\frac{\partial F_{1}}{\partial t} d t
$$

we obtain

$$
\frac{\partial F_{1}}{\partial t}=K-H, \quad p_{i}=\frac{\partial F_{1}}{\partial \bar{q}_{i}}, \quad-P_{i}=\frac{\partial F_{1}}{\partial \bar{Q}_{i}}
$$

$i=1, \ldots, N$. We call to (13) the canonical fractional transformations of first kind.

\subsection{Canonical fractional transformations of second kind}

We shall now introduce canonical transformations of second type with a generating function $F_{2}(t, \overline{\mathbf{q}}, \mathbf{P})$. We begin by noting that the transformation from $\bar{q}_{i}$ and $\bar{Q}_{i}$ to $\bar{q}_{i}$ and $P_{i}$ can be accomplished by a Legendre 
transformation. Indeed, by equations (13),$-P_{i}=\frac{\partial F_{1}}{\partial Q_{i}}$. This suggests that the generating function $F_{2}$ can be defined by

$$
F_{2}(t, \overline{\mathbf{q}}, \mathbf{P})=F_{1}(t, \overline{\mathbf{q}}, \overline{\mathbf{Q}})+\sum_{i=1}^{N} P_{i} \bar{Q}_{i}
$$

From equation (12) we obtain

$$
\begin{aligned}
\sum_{i=1}^{N} p_{i} \frac{d \bar{q}_{i}}{d t}-H(t, \mathbf{q}, \mathbf{p}) & -\sum_{i=1}^{N} P_{i} \frac{d \bar{Q}_{i}}{d t}+K(t, \mathbf{Q}, \mathbf{P}) \\
& =\frac{d}{d t}\left(F_{2}(t, \overline{\mathbf{q}}, \mathbf{P})-\sum_{i=1}^{N} P_{i} \bar{Q}_{i}\right) \\
& =\frac{d}{d t} F_{2}(t, \overline{\mathbf{q}}, \mathbf{P})-\sum_{i=1}^{N} P_{i} \frac{d \bar{Q}_{i}}{d t}-\sum_{i=1}^{N} \bar{Q}_{i} \frac{d P_{i}}{d t} .
\end{aligned}
$$

Hence,

$$
\left(\sum_{i=1}^{N} p_{i} \frac{d \bar{q}_{i}}{d t}-H\right) d t+\left(\sum_{i=1}^{N} \bar{Q}_{i} \frac{d P_{i}}{d t}+K\right) d t=d F_{2}(t, \overline{\mathbf{q}}, \mathbf{P})
$$

Repeating the procedure followed for $F_{1}$ in Section $\mathbf{3 . 3}$, we obtain the transformation equations

$$
\frac{\partial F_{2}}{\partial t}=K-H, \quad p_{i}=\frac{\partial F_{2}}{\partial \bar{q}_{i}}, \quad \bar{Q}_{i}=\frac{\partial F_{2}}{\partial P_{i}}, \quad i=1, \ldots, N .
$$

\subsection{Fractional Hamilton-Jacobi equation}

Under the assumption that $K$ is identically zero, for integer order derivatives we know, from equations (91), that the new coordinates are constant. The same situation occurs, under appropriate assumptions, in the fractional setting when in presence of Riemann-Liouville or Caputo derivatives [15, 22]. In our general combined setting this is not the case, in particular for the new momentum $P$. To solve the problem we proceed as follows. Assume that the transformed Hamiltonian, $K$, is identically zero. Then the equations of motion are

$$
\frac{\partial K}{\partial P_{i}}={ }^{C} D_{\gamma_{i}}^{\alpha_{i}, \beta_{i}} Q_{i}=0, \quad \frac{\partial K}{\partial Q_{i}}=D_{1-\gamma_{i}}^{\beta_{i}, \alpha_{i}} P_{i}=0, \quad i=1, \ldots, N .
$$

The function $K$ is related with the old Hamiltonian $H$ and the generating function $F$ by $K=H+\frac{\partial F}{\partial t}$. Hence, $K$ will be zero if, and only if, $F$ satisfies 
the equation $H(t, \mathbf{q}, \mathbf{p})+\frac{\partial F}{\partial t}=0$. It is convenient to take $F$ as a function of $\bar{q}_{i}$ and $P_{i}$. Then, from equations (14), we can write

$$
H\left(t, \mathbf{q}, \frac{\partial F_{2}}{\partial \overline{\mathbf{q}}}\right)+\frac{\partial F_{2}}{\partial t}=0 .
$$

Equation (15) is a fractional version of the Hamilton-Jacobi equation. Therefore, the quantum wave equation with the combined ${ }^{C} D_{\gamma}^{\alpha, \beta}$ fractional derivative is suggested to be

$$
H\left(t, \mathbf{q},-i \hbar \frac{\partial}{\partial \overline{\mathbf{q}}}\right) \psi=i \hbar \frac{\partial \psi}{\partial t},
$$

where $\psi$ is a wave equation.

\section{Conclusion}

In this paper we give an Hamiltonian formulation to the general fractional Euler-Lagrange equations obtained in [19]. Motivated by the recent results in the literature of Physics dealing with fractional mechanics and nonconservative systems [1, 15, 22, we illustrate some possible applications of our combined variational calculus in mechanics and quantization.

\section{Acknowledgments}

This work was supported by FEDER funds through COMPETE Operational Programme Factors of Competitiveness — and by Portuguese funds through the Center for Research and Development in Mathematics and Applications (University of Aveiro) and the Portuguese Foundation for Science and Technology (FCT), within project PEst-C/MAT/UI4106/2011 with COMPETE number FCOMP-01-0124-FEDER-022690. A.B. Malinowska was also supported by BUT grant S/WI/2/2011; and D.F.M. Torres by the FCT project PTDC/MAT/113470/2009.

\section{References}

[1] E.M.C. Abreu and C.F.L. Godinho, Fractional Dirac bracket and quantization for constrained systems. Phys. Rev. E 84 (2011), 026608, 8 pp.

[2] O.P. Agrawal, Formulation of Euler-Lagrange equations for fractional variational problems. J. Math. Anal. Appl. 272, No 1 (2002), 368-379.

[3] O.P. Agrawal, S.I. Muslih and D. Baleanu, Generalized variational calculus in terms of multi-parameters fractional derivatives. Commun. Nonlinear Sci. Numer. Simul. 16, No 12 (2011), 4756-4767.

[4] R. Almeida, S. Pooseh and D.F.M. Torres, Fractional variational problems depending on indefinite integrals. Nonlinear Analysis 75, No 3 (2012), 1009-1025. arXiv: 1102.3360

[5] R. Almeida and D.F.M. Torres, Leitmann's direct method for fractional optimization problems. Appl. Math. Comput. 217, No 3 (2010), 956-962. arXiv:1003.3088 
[6] R. Almeida and D.F.M. Torres, Necessary and sufficient conditions for the fractional calculus of variations with Caputo derivatives. Commun. Nonlinear Sci. Numer. Simul. 16, No 3 (2011), 1490-1500. arXiv:1007.2937

[7] R. Almeida and D.F.M. Torres, Fractional variational calculus for nondifferentiable functions. Comput. Math. Appl. 61, No 10 (2011), 3097-3104. arXiv:1103.5406

[8] D. Baleanu, Fractional Hamiltonian analysis of irregular systems. Signal Process. 86, No 10 (2006), 2632-2636.

[9] D. Baleanu, K. Diethelm, E. Scalas and J.J. Trujillo, Fractional Calculus: Models and Numerical Methods. World Scientific Publ. (Ser. on Complexity, Nonlinearity and Chaos, 3), N. Jersey-London-Singapore (2012).

[10] N.R.O. Bastos, R.A.C. Ferreira and D.F.M. Torres, Necessary optimality conditions for fractional difference problems of the calculus of variations. Discrete Contin. Dyn. Syst. 29, No 2 (2011), 417-437. arXiv:1007.0594

[11] G.S.F. Frederico and D.F.M. Torres, Nonconservative Noether's theorem in optimal control. Int. J. Tomogr. Stat. 5, No W07 (2007), 109-114. arXiv:math/0512468

[12] G.S.F. Frederico and D.F.M. Torres, Fractional conservation laws in optimal control theory. Nonlinear Dynam. 53, No 3 (2008), 215-222. arXiv:0711.0609

[13] G.S.F. Frederico and D.F.M. Torres, Fractional Noether's theorem in the RieszCaputo sense. Appl. Math. Comput. 217, No 3 (2010), 1023-1033. arXiv:1001.4507

[14] H. Goldstein, Classical Mechanics. Addison-Wesley Press, Inc., Cambridge, MA (1951).

[15] A.K. Golmankhaneh, Fractional Poisson bracket. Turk. J. Phys. 32 (2008), 241-250. arXiv:0807.4255

[16] M.A.E. Herzallah and D. Baleanu, Fractional-order Euler-Lagrange equations and formulation of Hamiltonian equations. Nonlinear Dynam. 58, No 1-2 (2009), 385391.

[17] M.A.E. Herzallah and D. Baleanu, Fractional-order variational calculus with generalized boundary conditions. Adv. Difference Equ. 2011 (2011), Article ID 357580, 9 pp.

[18] A.B. Malinowska and D.F.M. Torres, Leitmann's direct method of optimization for absolute extrema of certain problems of the calculus of variations on time scales. Appl. Math. Comput. 217, No 3 (2010), 1158-1162. arXiv:1001.1455

[19] A.B. Malinowska and D.F.M. Torres, Fractional calculus of variations for a combined Caputo derivative. Fract. Calc. Appl. Anal. 14, No 4 (2011), 523-537. arXiv: 1109.4664

[20] A.B. Malinowska and D.F.M. Torres, Multiobjective fractional variational calculus in terms of a combined Caputo derivative. Appl. Math. Comput. 218, No 9 (2012), 5099-5111. arXiv:1110.6666

[21] T. Odzijewicz, A.B. Malinowska and D.F.M. Torres, Fractional variational calculus with classical and combined Caputo derivatives. Nonlinear Anal. 75, No 3 (2012), 1507-1515. arXiv:1101.2932

[22] E.M. Rabei and B.S. Ababneh, Hamilton-Jacobi fractional mechanics. J. Math. Anal. Appl. 344, No 2 (2008), 799-805.

[23] E.M. Rabei, K.I. Nawafleh, R.S. Hijjawi, S.I. Muslih and D. Baleanu, The Hamilton formalism with fractional derivatives. J. Math. Anal. Appl. 327, No 2 (2007), 891897.

[24] F. Riewe, Nonconservative Lagrangian and Hamiltonian mechanics. Phys. Rev. E (3) 53, No 2 (1996), 1890-1899. 
[25] F. Riewe, Mechanics with fractional derivatives. Phys. Rev. E (3) 55, No 3 (1997), Part B, 3581-3592.

[26] C.J. Silva and D.F.M. Torres, Absolute extrema of invariant optimal control problems. Commun. Appl. Anal. 10, No 4 (2006), 503-515. arXiv:math/0608381

[27] D.F.M. Torres, On the Noether theorem for optimal control. Eur. J. Control 8, No 1 (2002), 56-63.

[28] D.F.M. Torres, Gauge symmetries and Noether currents in optimal control. Appl. Math. E-Notes 3 (2003), 49-57. arXiv:math/0301116

[29] D.F.M. Torres, Proper extensions of Noether's symmetry theorem for nonsmooth extremals of the calculus of variations. Commun. Pure Appl. Anal. 3, No 3 (2004), 491-500.

[30] D.F.M. Torres and G. Leitmann, Contrasting two transformation-based methods for obtaining absolute extrema. J. Optim. Theory Appl. 137, No 1 (2008), 53-59. arXiv:0704.0473

${ }^{1}$ Faculty of Computer Science Biatystok University of Technology 15-351 Biatystok, POLAND

e-mail: a.malinowska@pb.edu.pl Received: February 21, 2012

${ }^{2}$ Center for Research and Development in Mathematics and Applications Department of Mathematics, University of Aveiro 3810-193 Aveiro, PORTUGAL

e-mail:delfim@ua.pt 